\title{
Sub-pixel edge location in thermal images using a mean square measure
}

\author{
by R. Bąbka, W. Minkina
}

Institute of Electronics and Control Systems, Technical University of Częstochowa Al. Armii Krajowej 17, 42-200 Częstochowa, Poland

\begin{abstract}
In the paper a statistical method of edge location on the basis of the object radiation signal taken directly from thermogram has been presented. The proposed edge location method is based on maximum searching of mean square measure in an area. Considering the edge as an area between two isothermal surfaces, the maximum of variance in the edge neighbourhood can determine location of the edge. There was introduced a thermal edge model including such parameters as: spatial resolution, bias and a range of the edge temperatures.
\end{abstract}

\section{Introduction}

Algorithms of the edge location with sub-pixel precision are usually considered in case of visual images [1]. Due to limited spatial resolution, the loss of information about the signal edge must be reconstructed by means of a mathematical approximation model of the edge. A similar solution can be applied to the edge analysis of thermal objects registered using the infrared thermography technique [2,3]. The main problems in mathematical model of forming of the infrared edge are: errors of method of measurement $[4,5,7]$ as well as finite spatial resolution of the thermal camera [8-10]. In the paper a statistical method of edge location on the basis of the object radiation signal taken directly from thermogram has been presented. There was introduced a thermal edge model including such parameters as: spatial resolution, bias and a range of the edge temperatures.

\section{Edge model}

An ideal physical edge may be considered as the step function determined in the spatial domain. In fact, edge images received from optical system are disturbed by lens aberration and disperse effects. The former are responsible for shape changes in the image, the later are responsible for details recognition quality. Assuming sub-pixel precision of the edge location, especially the last influence of effect must be taken into account.

For visible optical systems as well as for infrared systems a degree of blurring is determined by Spread Response Function (SRF). In the one-dimensional case this function equals the Line Spread Function (LSF)[9]:

$$
L S F(x)=\exp \left(-\frac{x^{2}}{2 \cdot \sigma^{2}}\right)
$$

where $\sigma$ is the parameter of blurring degree (related to spatial resolution of the system). LSF determines one-dimensional spatial impulse response. So the step response of the system is:

$$
E S F(x)=\int L S F(x) d x
$$


where $\operatorname{ESF}(x)$ is the Edge Spread Function which describes edge signal received the by camera. Considering (1) and (2) the blurred edge model is expressed as:

$$
E S F(x, \sigma)=\sqrt{\frac{\pi}{2}} \cdot \sigma \cdot \operatorname{erf}\left(\frac{1}{\sqrt{2} \sigma} \cdot x\right)
$$

For thermal imaging, mainly for simulation purposes, more useful form of (3) is if $\mathrm{ESF}(\mathrm{x})$ represents radiation intensity $\mathrm{I}(\mathrm{x})$ and depends on temperature. Taking into account the well known formula [5]:

$$
I(T)=\frac{R}{\exp \left(\frac{B}{T}\right)-F}
$$

and introducing variables: $\mathrm{T}_{0}$ - bias temperature (lower temperature of the edge), $\Delta \mathrm{T}-$ temperature "height" of the edge, then substituting:

$$
\begin{aligned}
& I_{0}=I\left(T_{0}\right) \\
& \Delta I=I\left(T_{0}+\Delta T\right)-I\left(T_{0}\right)
\end{aligned}
$$

(3) takes form:

$$
\begin{aligned}
& \operatorname{EDGE}\left(x, \sigma, T_{0}, \Delta T\right)=\frac{R}{\exp \left(\frac{B}{T_{0}}\right)-F}+ \\
& +\left(\frac{R}{\exp \left(\frac{B}{T_{0}+\Delta T}\right)-F}-\frac{R}{\exp \left(\frac{B}{T_{0}}\right)-F}\right) \cdot \frac{1}{2} \cdot\left(1+\frac{2 E S F(x, \sigma)}{\sigma \sqrt{2 \pi}}\right)
\end{aligned}
$$

where $\mathrm{R}, \mathrm{B}, \mathrm{F}$ - calibration constants of a thermal camera.

Figure 1 presents result of edge modelling using (7) for 3 different $\sigma$ values and for $\mathrm{T}_{0}=293 \mathrm{~K}$ and $\Delta \mathrm{T}=60 \mathrm{~K}$

The last stage of modelling includes sampling process. For $1 \mathrm{D}$ case the pixel signal taken from the detector area is averaged according to:

$$
\operatorname{PIXELSIGNAL}(x, \sigma, \Delta x)=\frac{1}{\Delta x} \cdot \int_{x-\frac{\Delta x}{2}}^{x+\frac{\Delta x}{2}} E D G E(\tau, \sigma) d \tau
$$

where, $\Delta \mathrm{x}$ - denotes pixel dimension.

Assuming $\Delta x=1, x$ scale both for continuous discrete signals remains unchanged. Figure 2 shows graphic interpretation of the final discrete one-dimensional edge model for $\sigma=0.5$. 


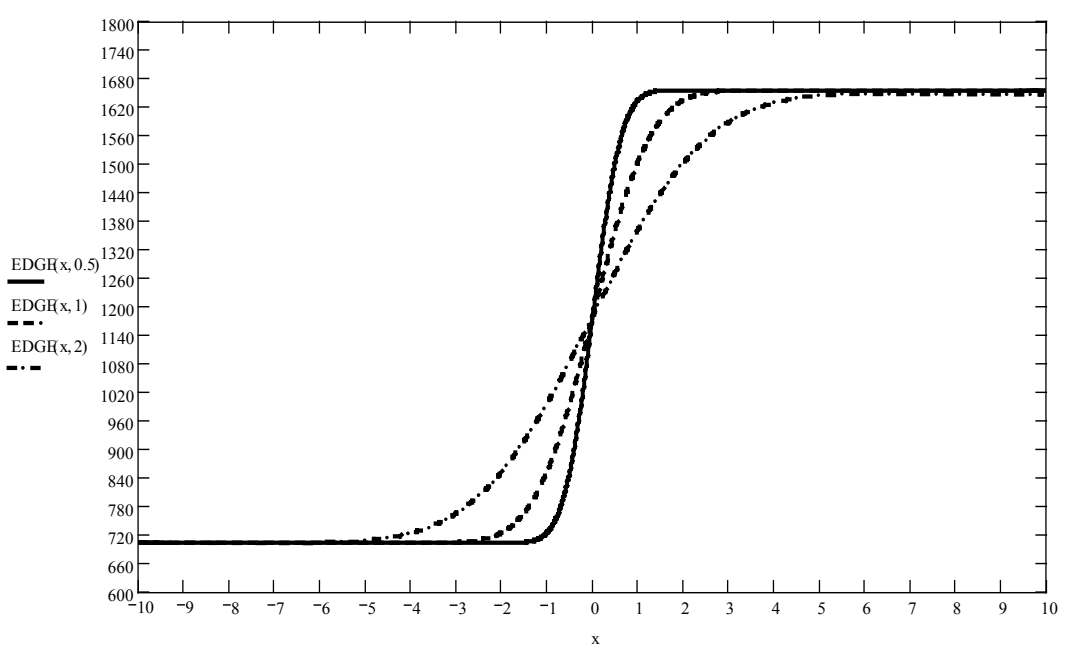

Fig. 1. Edge model (intensity object signal) for different values of $\sigma$

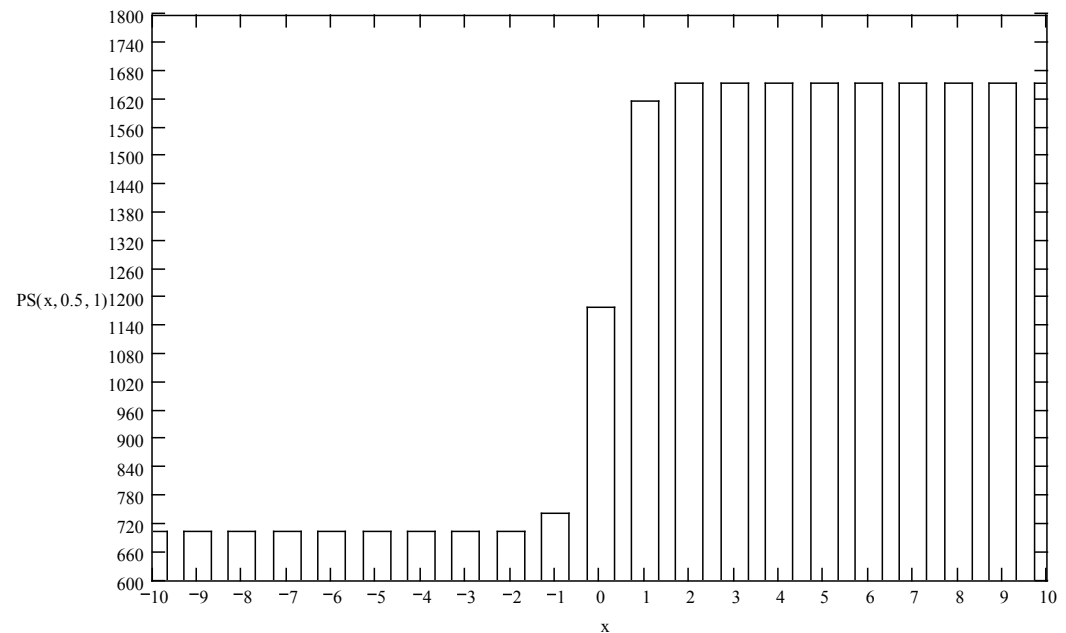

Fig. 2. Discrete edge model. Bars denotes pixel signal values

\section{Sub-pixel edge searching}

The proposed method is based on maximum searching of mean square measure in an edge neighbourhood area. Figure 3 presents a principle of searching for one-dimensional ideal edge location. The edge discriminates two areas having values a and $-a$ respectively.

The model presented above can be expressed by binomial distribution. Frequency of a in window area $\left(x_{0}, x_{2}\right)$ is given by the formula:

$$
P(Y=a)=P\left[X \in\left(x_{0}, x_{2}\right)\right]=p=\frac{x_{2}-x_{0}}{x_{2}-x_{1}}
$$


a)

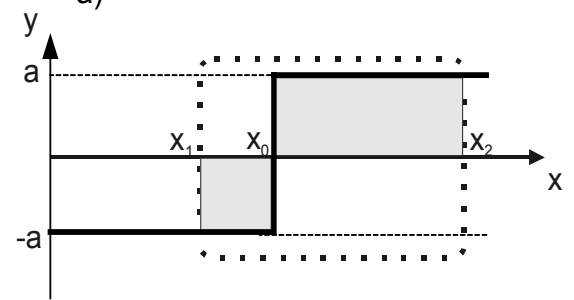

b)

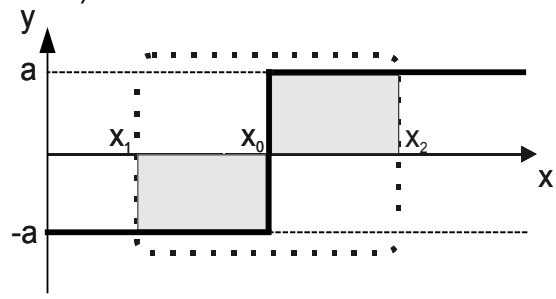

Fig. 3. Edge location searching using mean square measure - statistical approach: (dotted area denotes a moving data analysis window across the edge),

a) frequency of $a$ in data window is bigger than frequency of $-a$,

b) frequencies of $a$ and -a in data window are the same

For the considered distribution, variance in $\left(x_{1}, x_{2}\right)$ equals [10]:

$$
V(y)=4 a^{2} p(1-p)=4 a^{2}\left(p-p^{2}\right)
$$

and achieves maximum for

$$
p=0.5 \Rightarrow \quad x_{0}-x_{1}=x_{2}-x_{0}
$$

which determinates co-ordinate of the edge. Relationships presented above can be a proof of thesis, that the maximum of variance in the considered edge neighbourhood can determine location of the edge. Since the co-ordinate of this maximum is not depended on $y$ so the bias error related to measurement of $y$ may be eliminated. In practice, for $V(x)$ estimation the following expressions can be used:

$$
\begin{aligned}
& \operatorname{VPS}(N, x)=\frac{1}{N-1} \cdot \sum_{i=-\frac{N}{2}}^{\frac{N}{2}-1}\left(P S M\left(x-i \cdot \Delta x-\frac{\Delta x}{2}\right)-P S M_{a v}(N, x)\right)^{2} \\
& \operatorname{PSM}_{a v}(N, x)=\frac{1}{N} \cdot \sum_{i=-\frac{N}{2}}^{\frac{N}{2}-1} P S M\left(x-i \cdot \Delta x-\frac{\Delta x}{2}\right)
\end{aligned}
$$

for even number of samples, or for odd number of samples:

$$
\begin{aligned}
& \operatorname{VPS}(N, x)=\frac{1}{N-1} \cdot \sum_{i=-\frac{(N-1)}{2}}^{\frac{N-1}{2}}\left(P S M(x-i \cdot \Delta x)-P S M_{a v}(N, x)\right)^{2} \\
& \operatorname{PSM}_{\mathrm{av}}(N, x)=\frac{1}{N} \cdot \sum_{i=-\frac{(N-1)}{2}}^{\frac{N-1}{2}} P S(x-i \cdot \Delta x)
\end{aligned}
$$

where: $\mathrm{N}$ - number of samples in data window (corresponding to fig. 3 ), $\Delta \mathrm{x}$ - pixel dimension (corresponding to fig. 2 and equation (8)), PSM(x)=PIXELSIGNAL( $x$ ) (equation (8)), $\mathrm{PSM}_{\mathrm{av}}$ - average of $\mathrm{N}$ pixel samples, VPS - variance estimator of $\mathrm{N}$ samples. 
Since it was assumed $\Delta x=1$, the corresponding co-ordinate $x$ of VPS $(x)$ and the integer $\mathrm{i}$-th value VPS $\mathrm{S}_{\mathrm{i}}$ matches each other $\left(\mathrm{x}_{\mathrm{i}}=\mathrm{i}\right)$. In this case the edge location $\mathrm{X}_{0}$ is estimated by:

$$
X_{0}(M, V P S)=\sum_{i=0}^{M} \frac{x_{i} \cdot V P S_{l}}{\sum_{l=0}^{M} V P S_{l}}
$$

where $(M+1)$ is a number of samples in $X_{0}$ neighbourhood, which exceed VPS estimation error calculated from [6]:

$$
u_{k}=\sqrt{\frac{1}{N} \cdot\left(\xi_{4}-\frac{N-3}{N-1} \cdot \sigma^{4}\right)}
$$

where $\sigma$ is the standard deviation of measurement using thermal camera, $\xi_{4}-4$-th order moment of thermal field registered in isothermal part of the edge signal.

The measurement error of $X_{0}$ can be obtained from the error propagation theory:

$$
E R R=\sqrt{\sum_{k=0^{\prime}}^{M}(D V P S(k))^{2} \cdot\left(u_{k}\right)^{2}}
$$

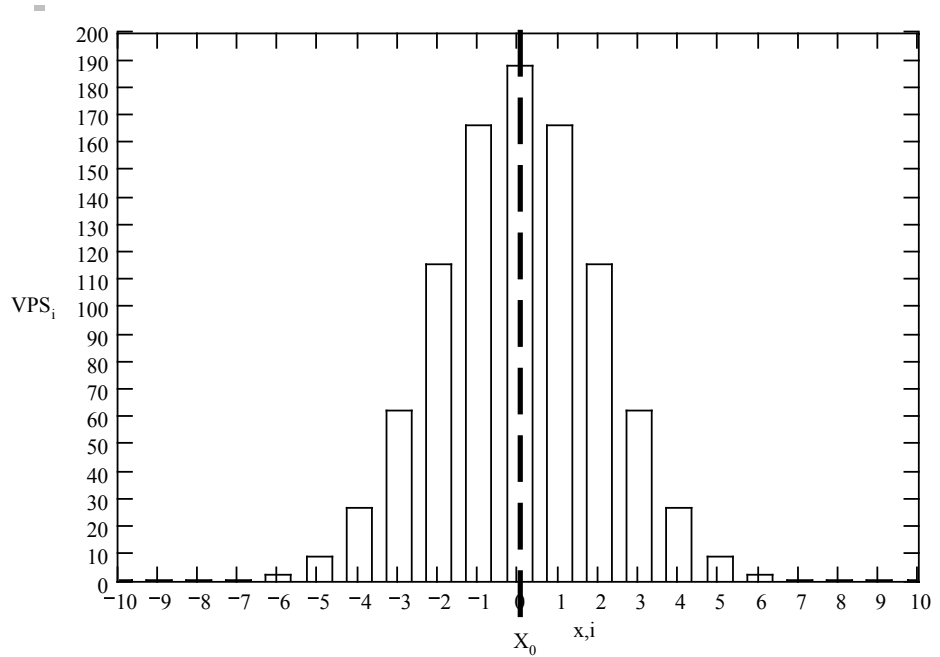

Fig. 4. Graphical interpretation of edge location according to equation (16) for $M=12$

where

$$
\operatorname{DVPS}(k)=\frac{\partial}{\partial V P S_{k}}(X(N, V P S)) \quad \operatorname{DVPS}(k)=\frac{x_{k}}{\sum_{i=0}^{M} V P S_{i}}-\sum_{j=0}^{M} \frac{x_{j} \cdot V P S_{j}}{\left(\sum_{i=1}^{M} V P S_{i}\right)^{2}}
$$

and $u_{k}$ is determined by equation (17). 


\section{Simulations and experiment - results}

On the basis of the presented edge model, simulation tests and experiments have been conducted. During the simulation tests, there were considered different levels of noise. The results are presented in table 1.

The laboratory stand for measurements was equipped with thermal camera ThermaCAM PM 595 (FLIR) and lenses $24^{\circ} \times 18^{\circ}$ Figure 5 explains the method of thermogram analysis. As a thermal object a radiator $50 \times 50 \mathrm{~mm}( \pm 0.1 \mathrm{~mm})$ has been used.

Table 1. $\delta_{\text {loc }}$ - location error related to pixel dimension,

$\delta_{\text {disl }}-$ dislocation error related to pixel dimension.

\begin{tabular}{|c|c|c|c|c|c|c|c|c|c|}
\hline & \multicolumn{8}{|c|}{ Number of samples - $\mathrm{N}$} \\
\hline & & \multicolumn{2}{|c|}{4} & \multicolumn{2}{|c|}{6} & \multicolumn{2}{|c|}{8} & \multicolumn{2}{|c|}{10} \\
\hline & & $\delta_{l o c} \%$ & $\delta_{\text {disl }} \%$ & $\delta_{l o c} \%$ & $\delta_{\text {disl }} \%$ & $\delta_{\text {loc }} \%$ & $\delta_{\text {disl }} \%$ & $\delta_{l o c} \%$ & $\delta_{\text {disl }} \%$ \\
\hline \multirow{3}{*}{$\begin{array}{l}\frac{0}{10} \\
\frac{1}{2}\end{array}$} & 0 & 23 & 2.3 & 15 & 1.6 & 12 & 1 & 3 & 1 \\
\hline & $0.1 \mathrm{~N}(0,1)$ & 22 & 5.6 & 4.2 & 2.1 & 4.3 & 1.6 & 3 & 1.7 \\
\hline & $1 \mathrm{~N}(0,1)$ & 120 & 5.6 & 62 & 3.2 & 5.4 & 2.6 & 56 & 2.4 \\
\hline
\end{tabular}
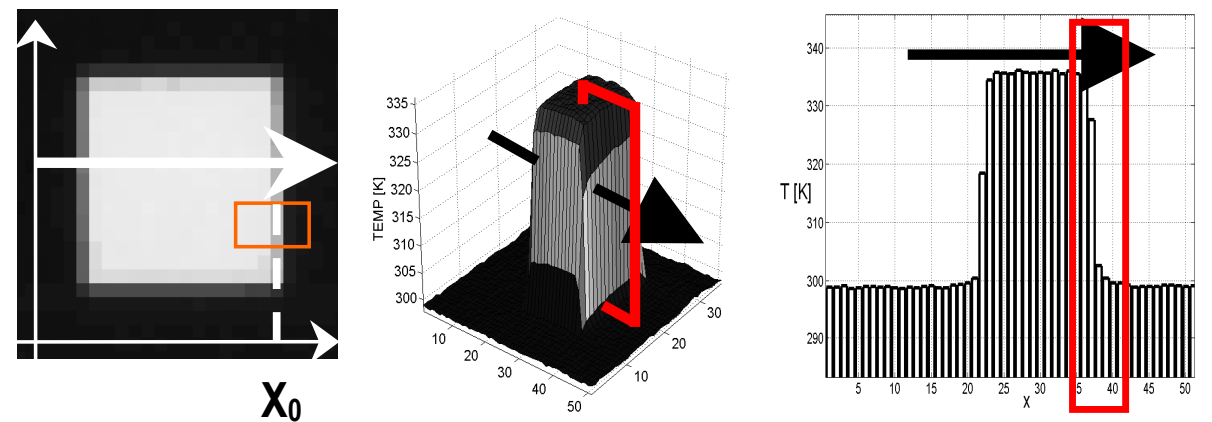

Fig. 5. Edge location analysis on the basis of radiator thermogram

There were conducted 216 measurements collected at distances: 0.5, 1 and 2 meters. The standard deviation of measurements did not exceed 0.02 of pixel. The width of the radiator received from experiment was biased up to 0.2 of the pixel dimension. This error was caused probably by geometrical distortion error of optical system since it was getting smaller with increasing the distance form camera to object.

\section{REFERENCES}

[1] Boon G.W Shan Y., Sub-Pixel Location of Edges With Non-Uniform Blurring: A Finite Closed-Form Approach. Image and Vision Computing, 18(2000) p. 1015-1023.

[2] Bąbka R., Minkina W., Simulation Tests of Mean Square Measure Usability for a Thermal Field Analysis Registered Using Termovision System, Proceedings XI Symposium on Measuring Systems - Modelling and Simulation", Krynica, 1721.09.2001, p.129-136. (in polish). 
[3] Babka R., Minkina W., Wild W., Evaluation of Mean Square Measure Application to Edge Detection of Objects in Thermal Images, Pomiary Automatyka Kontrola (PAK) 68(2001) №11p. 9-12 (in polish).

[4] Chrzanowski K., Non-Contact Thermometry, Measurements Errors. SPIE PL, Research and Development Treatises, Vol. 7 Warsaw 2000.

[5] FLIR, Research \& Development (R\&D) Level II -2000. Infrared Training Course International (itc-i).

[6] Guide to Expression of Uncertainty in Measurement, ISO 1995.

[7] Minkina W., Rutkowski P., Wild W., Thermovision Measurement Basics, Pomiary Automatyka Kontrola (PAK) 46(2000) №1, part I - p. 7-10 and part II - p. 11-14 (in polish).

[8] Minkina W., Thermovision Measurement Basics, Pomiary Automatyka Kontrola (PAK) 68(2001) №11, part III - p. 5-8 (in polish).

[9] Multi-author work edited by Gerlach G., Technisches Messen 3/1999 pp. 35, 9/1999 pp. 49

[10] Ott L., An Introduction to Statistical Methods and Data Analysis; Doxbury Press, Boston 1984

[11] Woźnicki J., Podstawowe techniki przetwarzania obrazu; WKŁ, Warszawa 1996. 